Revista Brasileira de

Engenharia Agrícola e Ambiental

v.15, n.10, p.1037-1045, 2011

Campina Grande, PB, UAEA/UFCG - http://www.agriambi.com.br

agriambi

Protocolo 078.10 - 26/04/2010 • Aprovado em 09/08/2011

\title{
Fluxo de calor sensível em cultivo de feijão-caupi
}

\author{
José R. de S. Lima', Antonio C. D. Antonino ${ }^{2}$, Carlos A. B. de 0. Lira ${ }^{2}$, \\ Willames de A. Soares ${ }^{3} \&$ Eduardo S. de Souza ${ }^{4}$
}

\begin{abstract}
RESU M O
0 presente trabalho objetivou comparar o fluxo de calor sensível obtido pelo método do balanço de energia-razão de Bowen (H_B) e pelo método aerodinâmico (H_A), em uma área de 4 ha cultivada com feijão-caupi em Areia-PB ( $\overline{6}^{\circ} 58^{\prime} 12^{\prime \prime} \mathrm{S}$ e $35^{\circ} 42^{\prime} 15^{\prime \prime} 0,620$ m). Foram coletados dados de saldo de radiação, fluxo de calor no solo, temperatura e umidade do solo, temperatura e umidade relativa do ar, velocidade do vento e precipitação pluvial, os quais eram lidos a cada minuto, e armazenados, em uma central de aquisição de dados, como médias a cada $30 \mathrm{~min}$, com exceção da pluviometria, cujo valor foi totalizado no período. O bteve-se excelente concordância entre $\mathrm{H}$ A e H B, com um índice "d" de 0,980, um erro padrão (EP) de $14,88 \mathrm{~W} \mathrm{~m}^{-2}$, e sem diferença significativā entre- ${ }_{\mathrm{H}} \_\mathrm{A}$ e $\mathrm{H}_{-} \mathrm{B}$, pelo teste t-Student. Deste modo, o método aerodinâmico pode ser usado em conjunto com medidas de saldo de radiação e de fluxo de calor no solo, para se estimar o fluxo de calor latente e, consequentemente, a evapotranspiração da cultura do feijão-caupi, para as condições edafoclimáticas do Brejo Paraibano.
\end{abstract}

Palavras-chave: balanço de energia, razão de Bowen, método aerodinâmico, evapotranspiração

\section{Sensible heat flux in cowpea}

\begin{abstract}

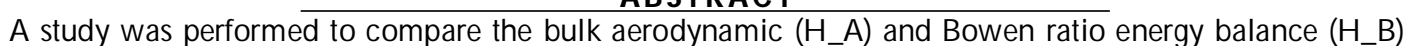
methods in the estimation of the sensible heat flux in a cowpea crop. D ata of net radiation, soil heat flux, soil water content, soil temperature, air temperature, relative humidity, wind speed and rainfall were collected on 30-min time intervals in an area of 4 ha belonging to the municipality of Areia, PB $\left(6^{\circ} 58^{\prime} 12^{\prime \prime}\right.$ $\mathrm{S} \mathrm{e} 35^{\circ} 42^{\prime} 15^{\prime \prime} \mathrm{W}, 620 \mathrm{~m}$ ). Results showed that there was a good agreement between $\mathrm{H}$ A and $\mathrm{H} B$ with an index " $\mathrm{d}$ " of 0.98 and standard error of estimate (SEE) of $14.88 \mathrm{~W} \mathrm{~m}^{-2}$, no significant difference between $\mathrm{H}_{-}$A H_B $\mathrm{H}_{-}$, by the t-Student test. Thus, the bulk aerodynamic method can be used in conjunction with measurements of net radiation and soil heat flux to estimate the evapotranspiration of the cowpea under soil and climatic condition of the "Brejo Paraibano".
\end{abstract}

Key words: energy balance, Bowen ratio, aerodynamic method, evapotranspiration

${ }^{1}$ U FRPE/U AG, Av. Bom Pastor S/N, Boa Vista, CEP 55296-901, Garanhuns, PE. Fone (87) 3761-0882. E-mail: romualdo@uag.ufrpe.br 2 U FPE/D EN, Av. Prof. Luís Freire 1000, Cidade U niversitária, CEP 50740-540, Recife, PE. Fone (81) 2126-7973. E-mail: acda@ufpe.br; cabol@ufpe.br ${ }^{3}$ FACETEG/U PE. Rua Cap. Pedro Rodrigues, 105, Brasília, CEP 55.294-902, Garanhuns, PE. Fone (87) 3761-8210. E-mail: willamess@yahoo.com.br ${ }_{4}^{4}$ U FRPE/U AST, Fazenda Saco, S/N , CP 063, Zona Rural, CEP 56.900-000, Serra Talhada, PE. Fone (87)3831-2053. E-mail: edu souza_pe@yahoo.com.br 


\section{INTRODUÇÃO}

A quantificação dos componentes do balanço de energia é essencial para o planejamento dos recursos hídricos de uma área, para estimar o fornecimento potencial de água e compreender os efeitos ecológicos decorrentes do desenvolvimento das regiões (Bidlake et al., 1996) e para o planejamento da irrigação das culturas, visando a uma compreensão melhor e modelagem dos processos de troca de massa e de energia que ocorrem na superfície da terra, especialmente em relação às mudanças climáticas, quando então pequenas mudanças no balanço global de energia podem resultar em impactos drásticos na biosfera (Gentine et al., 2007; Zhou \& Zhou, 2009).

Além disso, uma compreensão melhor desses processos em áreas agrícolas, permite também uma avaliação melhor da evapotranspiração e fotossíntese, as quais afetam diretamente a acumulação de biomassa e a produtividade dos cultivos (Rolim et al., 2008).

O balanço de energia pressupõe que o saldo de radiação $(\mathrm{Rn})$ na superfície da terra se reparte em fluxo de calor para o solo (G), fluxo de calor sensível para o ar $(\mathrm{H})$, fluxo de calor latente de evaporação (LE), fotossíntese e trocas devidas às atividades metabólicas e ao armazenamento nos tecidos das plantas. Considerando que os dois últimos componentes são, em geral, inferiores ao erro experimental na medida dos componentes principais, o balanço de energia considera, geralmente, apenas os três primeiros processos (Bergamaschi et al., 1988).

O saldo de radiação $(\mathrm{Rn})$ e o fluxo de calor no solo $(\mathrm{G})$ podem ser medidos facilmente por meio de instrumentos. Entretanto, os fluxos de calor sensível (H) e de calor latente (LE) são de difícil medição direta, sendo que existem três métodos utilizados para a obtenção desses fluxos, quais sejam: o método do balanço de energia razão de Bowen, o método aerodinâmico e o método da correlação dos turbilhões (Lima, 2004).

O método do balanço de energia, razão de Bowen, é considerado um método padrão nas estimativas de $\mathrm{H}$ e LE, o qual vem sendo usado por diversos pesquisadores (Lima et al., 2005; Azevedo et al., 2007; Zhang et al., 2007; Neves et al., 2008; Souza et al., 2008; Teixeira et al., 2008; Bezerra et al., 2010; Silva et al., 2011).

O método aerodinâmico, baseado nas relações fluxogradiente, também vem sendo usado para a estimativa dos fluxos de calor sensível e de calor latente (Pieri \& Fuchs, 1990; Kolle, 1996; Polonio \& Soler, 2000; Rolim et al., 2008).

Ortega-Farias et al. (1996) utilizaram os métodos aerodinâmico e do balanço de energia razão de Bowen, para avaliar a variação do fluxo de calor sensível $(\mathrm{H})$ em grama sob diferentes condições de umidade volumétrica do solo e encontraram uma excelente concordância entre os métodos na estimativa de $\mathrm{H}$.

A principal vantagem no uso do método aerodinâmico é evitar as medições dos gradientes de umidade, já que os gradientes de temperatura do ar são obtidos mais facilmente e mais precisos para a estimativa do fluxo de calor sensível, combinado com o balanço de energia para a determinação do fluxo de calor latente (Rana \& Katerji, 2000).
Em face ao exposto, o trabalho teve como objetivo comparar os métodos do balanço de energia razão de Bowen e aerodinâmico na determinação do fluxo de calor sensível, para todo o ciclo fenológico de uma cultura de feijão-caupi e para dois curtos períodos deste ciclo, com condições contrastadas de umidade na camada superficial do solo.

\section{Material e mÉTOdos}

As medidas necessárias para a estimativa do fluxo de calor sensível pelos métodos aerodinâmico e balanço de energia razão de Bowen foram efetuadas em uma área de 4,0 ha, localizada na Fazenda Chã de Jardim, pertencente ao Centro de Ciências Agrárias, da Universidade Federal da Paraíba, situada na microrregião do Brejo Paraibano, no município de Areia, Estado da Paraíba (60 58' 12' S e 35 42' 15" O, 620 m de altitude). O clima na região, pela classificação de Köppen, é do tipo As' (quente e úmido). De acordo com dados da Estação Meteorológica do Centro de Ciências Agrárias da Universidade Federal da Paraíba, a precipitação média anual no município de Areia é de $1.400 \mathrm{~mm}$; a temperatura média anual, de $24,5^{\circ} \mathrm{C}$ e a umidade relativa média, de $80 \%$. O quadrimestre mais chuvoso - constituído dos meses de abril, maio, junho e julho - representa $62 \%$ do total médio anual (Oliveira et al., 2009). O solo da área é classificado como Latossolo Amarelo (EMBRAPA, 2006).

Os dados coletados incluíram temperatura do ar (Ta), umidade relativa (UR), velocidade do vento (u), saldo de radiação $(\mathrm{Rn})$, fluxo de calor no solo $(\mathrm{G})$, temperatura do solo (Ts), umidade volumétrica do $\operatorname{solo}(\theta)$ e precipitação pluvial $(\mathrm{P})$.

A temperatura do ar, a umidade relativa e a velocidade do vento, foram medidas em dois níveis $\left(\mathrm{z}_{1}=0,35 \mathrm{~m} \mathrm{e}_{2}=1,05 \mathrm{~m}\right)$ acima do dossel do feijão-caupi, os quais foram estabelecidos de acordo com a área de bordadura ou "fetch". Nesta pesquisa, o fetch variou de 100:1 a 141:1, ou seja, a espessura da zona a fluxo conservativo variou de $1,00 \mathrm{~m}$ a $1,41 \mathrm{~m}$, sendo que as medidas dos componentes do balanço de energia estavam dentro dessa faixa, uma vez que o maior nível de medição foi de $1,05 \mathrm{~m}$ (Lima, 2004). A temperatura e a umidade relativa foram medidas através de dois sensores HMP45C da Campbell Scientific Inc. A velocidade do vento foi medida usando-se dois anemômetros com velocidade de partida de $0,45 \mathrm{~m} \mathrm{~s}^{-1}$ (014A; Met One, Inc., Grants Pass, OR). O saldo de radiação foi medido por um radiômetro tipo Fritschen (Q7; REBS, Bellevue, WA). O fluxo de calor no solo foi medido por dois fluxímetros (HFT3; Campbell Scientific, Logan, UT) instalados em uma profundidade de $0,05 \mathrm{~m}$, além de duas sondas de temperatura do solo (108; Campbell Scientific Inc., Logan, UT), instaladas nas profundidades de 0,02 e $0,08 \mathrm{~m}$, respectivamente, e de um sensor de umidade volumétrica do solo (CS 615; Campbell Scientific Inc., Logan, UT) instalado numa profundidade de $0,05 \mathrm{~m}$. A precipitação pluvial foi medida por um pluviógrafo tipo báscula (TE525MM; Texas Electronics, Inc., Dallas, TX). Todas as medidas citadas acima foram amostradas a cada $60 \mathrm{~s}$ e armazenadas em um sistema de aquisição de dados CR 10X, da Campbell Scientific, como média a cada $30 \mathrm{~min}$, com exceção da precipitação cujo valor foi totalizado neste período. 
O período de coleta dos dados foi desde a semeadura (13/ 03/2002) até a colheita (14/06/2002) da cultura. O plantio foi realizado manualmente, por meio de matracas, no espaçamento de $1,0 \times 0,5 \mathrm{~m}$, sendo colocadas duas sementes por cova da cultivar "corujinha". Durante o ciclo fenológico da cultura não se detectou a presença de pragas nem a ocorrência de doenças. Foi efetuada uma adubação em fundação a qual constou de $100 \mathrm{~kg} \mathrm{ha}^{-1}$ de sulfato de amônia, $200 \mathrm{~kg} \mathrm{ha}^{-1}$ de cloreto de potássio e $178 \mathrm{~kg} \mathrm{ha}^{-1}$ de superfosfato triplo. O experimento foi realizado em condições de sequeiro tendo ocorrido um total de chuvas de 410,3 mm. A produtividade de grãos foi de $813 \mathrm{~kg} \mathrm{ha}^{-1}$ (Lima, 2004).

A comparação dos métodos do balanço de energia razão de Bowen e aerodinâmico na determinação do fluxo de calor sensível, foi realizada para todo o ciclo fenológico da cultura e para dois curtos períodos de oito dias, deste ciclo, com diferentes condições de umidade da camada superficial do solo, denominados "Período I" e "Período II". Os períodos I e II foram selecionados, em que a umidade volumétrica média na camada de $0-0,05 \mathrm{~m}$ foi de $0,20 \mathrm{~m}^{3} \mathrm{~m}^{-3}$ e de $0,13 \mathrm{~m}^{3} \mathrm{~m}^{-3}$, respectivamente. O Período I foi dos 11-18 dias após o plantio ( 23 a 30/03/2002), enquanto o Período II foi dos 27-34 dias após o plantio (08 a 15/04/2002). A precipitação pluvial total durante o Período I foi de 96,2 mm enquanto no Período II foi de 10,6 $\mathrm{mm}$.

O fluxo de calor no solo foi medido a $0,05 \mathrm{~m}$ de profundidade porém se necessita dos valores na superfície do solo. Deste modo, o fluxo de calor na superfície do solo $(\mathrm{G})$, foi dado por (Kustas et al., 2000):

$$
\mathrm{G}=\mathrm{G}_{\mathrm{p}}+\frac{\Delta \mathrm{T}_{\mathrm{S}} \mathrm{CD}}{\mathrm{t}}
$$

sendo:

$\mathrm{G}_{\mathrm{p}}$ - medição do fluxo de calor pelo fluxímetro, $\mathrm{W} \mathrm{m}^{-2}$

$\Delta \mathrm{T}_{\mathrm{S}}$ - variação na temperatura média do solo $\left({ }^{\circ} \mathrm{C}\right)$ durante o período de medição

$\mathrm{C}$ - capacidade térmica volumétrica do solo, $\mathrm{MJ} \mathrm{m}^{-3}{ }^{\circ} \mathrm{C}^{-1}$

D - profundidade do fluxímetro, $m$

t - duração do período de medição, s

$\mathrm{O}$ valor de $\mathrm{C}$ foi estimado somando-se as capacidades caloríficas dos vários constituintes do solo, ponderados de acordo com suas frações de volume, com base em Vries (1966):

$$
\mathrm{C}=1,92 \mathrm{f}_{\mathrm{m}}+2,51 \mathrm{f}_{0}+4,18 \theta
$$

sendo:

$\begin{array}{ll}\mathrm{f}_{\mathrm{m}} & \text { - fração de volume dos minerais }(0,47) \\ \mathrm{f}_{0} & \text { - fração de volume da matéria orgânica }(0,03) \\ \theta & \text { - umidade volumétrica do solo }\end{array}$

A umidade volumétrica utilizada foi a obtida, a cada $30 \mathrm{~min}$, pelos sensores instalados na profundidade de $0,05 \mathrm{~m}$.

O balanço de energia na superfície do solo pode ser escrito por meio da seguinte equação:

$$
\mathrm{Rn}=\mathrm{G}+\mathrm{H}+\mathrm{LE}
$$

em que:

$$
\begin{aligned}
& \mathrm{Rn} \text { - saldo de radiação, } \mathrm{W} \mathrm{m}^{-2} \\
& \mathrm{G} \text { - fluxo de calor no solo, } \mathrm{W} \mathrm{m}^{-2} \\
& \mathrm{H} \text { - fluxo de calor sensível, } \mathrm{W} \mathrm{m}^{-2} \\
& \mathrm{LE} \text { - fluxo de calor latente, } \mathrm{W} \mathrm{m}^{-2}
\end{aligned}
$$

A partição da energia disponível (Rn-G) entre fluxo de calor latente e fluxo de calor sensível, pode ser obtida pelo método do balanço de energia com base na razão de Bowen, obtida com as diferenças na temperatura do ar e na pressão de vapor, em dois níveis:

$$
\beta=\frac{\mathrm{H}}{\mathrm{LE}}=\gamma \frac{\Delta \mathrm{T}}{\Delta \mathrm{e}}
$$

em que:

$$
\begin{aligned}
& \gamma \quad \text { - constante psicrométrica, } 0,066 \mathrm{kPa}^{\circ} \mathrm{C}^{-1} \\
& \Delta \mathrm{T} \text { - diferença de temperatura do ar, }{ }^{\circ} \mathrm{C} \\
& \Delta \mathrm{e} \text { - diferença da pressão de vapor, } \mathrm{kPa}
\end{aligned}
$$

A partir das medições do saldo de radiação $(\mathrm{Rn})$, do fluxo de calor no solo $(\mathrm{G})$ e das diferenças de temperatura do ar $(\Delta \mathrm{T})$ e da pressão de vapor $(\Delta \mathrm{e})$, juntamente com a equação simplificada do balanço de energia (Eq. 3) e se utilizando a razão de Bowen ( $\beta$ ) (Eq. 4), procedeu-se ao cálculo do fluxo de calor sensível (H_B) segundo a equação:

$$
\mathrm{H}_{-} \mathrm{B}=\frac{\beta}{1+\beta}(\mathrm{Rn}-\mathrm{G})
$$

O fluxo de calor sensível pelo método aerodinâmico (H_A) foi obtido utilizando-se a seguinte equação:

$$
\mathrm{H}_{-} \mathrm{A}=\mathrm{c}_{\mathrm{p}} \rho_{\mathrm{a}} \Delta \mathrm{T} / \mathrm{r}_{\mathrm{a}}
$$

sendo:

$c_{p}$ - calor específico do ar em pressão constante, $\mathrm{J} \mathrm{kg}^{-1}{ }^{\circ} \mathrm{C}^{-1}$

$\rho_{\mathrm{a}}$ - densidade do ar, $\mathrm{kg} \mathrm{m}^{-3}$

$\Delta \mathrm{T}$ - diferença de temperatura do ar, ${ }^{\circ} \mathrm{C}$

$r_{a}$ - resistência aerodinâmica, $\mathrm{s} \mathrm{m}^{-1}$

A resistência aerodinâmica foi obtida pela equação:

$$
r_{a}=\left[\ln \left(\frac{\left(z_{2}-d\right)}{\left(z_{1}-d\right)}\right)-\psi h_{2}+\psi h_{1}\right] / k u_{*}
$$

em que:

os subscritos 1e 2 se referem aos níveis de medida

z - altura de medição da velocidade do vento e da temperatura do ar, $\mathrm{m}$

d - altura de deslocamento do plano zero, $\mathrm{m}$

k - constante de von Kárman $(0,41)$ 
$\psi h$ - função de correção de estabilidade para o transporte de calor

$$
\mathrm{u}_{*} \text { - velocidade de fricção }
$$

A altura de deslocamento do plano zero (d) foi obtida da altura da cultura $\left(\mathrm{h}_{\mathrm{c}}\right)$ como (Brutsaert, 1982):

$$
\mathrm{d}=\frac{2}{3 \mathrm{~h}_{\mathrm{c}}}
$$

A velocidade de fricção $\mathrm{u}_{*}$ foi obtida por:

$$
\mathrm{u}_{*}=\frac{\left(\mathrm{u}_{2}-\mathrm{u}_{1}\right) \mathrm{k}}{\ln \left(\frac{\left(\mathrm{z}_{2}-\mathrm{d}\right)}{\left(\mathrm{z}_{1}-\mathrm{d}\right)}\right)}-\psi \mathrm{m}_{2}+\psi \mathrm{m}_{1}
$$

sendo:

"u" - velocidade do vento

$\psi \mathrm{m}$ - função de correção de estabilidade para transporte de momentum

A correção da estabilidade da atmosfera é considerada por intermédio do comprimento de Monin-Obukhov, L, dado por:

$$
\mathrm{L}=-\frac{\rho_{\mathrm{a}} \mathrm{u}_{*}^{3}}{\operatorname{kg}\left(\frac{\mathrm{H}}{\overline{\mathrm{T}} \mathrm{c}_{\mathrm{p}}}\right)}
$$

sendo:

$$
\begin{aligned}
& \text { “g” - aceleração da gravidade } \\
& \mathrm{H} \text { - fluxo de calor sensível } \\
& \mathrm{T} \text { - temperatura do ar }
\end{aligned}
$$

A formulação para a correção da estabilidade da atmosfera usada neste trabalho foi aquela proposta por Dyer \& Hicks (1970):

$$
\begin{aligned}
& \phi_{\mathrm{m}}=(1-16 \zeta)^{-0.25} \\
& \phi_{\mathrm{h}}=(1-16 \zeta)^{-0.50}
\end{aligned}
$$

sendo:

$$
\begin{aligned}
& \zeta-(\mathrm{z}-\mathrm{d}) \mathrm{L}^{-1} \\
& \phi_{\mathrm{m}} \text { e } \phi_{\mathrm{h}} \text { - correções para momentum e calor, respectivamente }
\end{aligned}
$$

Deste modo, pode-se estimar $\psi_{\mathrm{m}}$ e $\psi_{\mathrm{h}}$ pelas seguintes expressões (Paulson, 1970):

$$
\begin{gathered}
\psi_{\mathrm{m}}=2 \ln [(1+\mathrm{x}) / 2]+\ln \left[\left(1+\mathrm{x}^{2}\right) / 2\right\rfloor-2 \arctan (\mathrm{x})+\pi / 2 \\
\psi_{\mathrm{h}}=2 \ln \left[\left(1+\mathrm{x}^{2}\right) / 2\right\rfloor
\end{gathered}
$$

sendo:

$$
\mathrm{x}-1 / \phi_{\mathrm{m}}
$$

Tendo em vista que as funções de correção da estabilidade dependem de $\mathrm{u}_{*}$ e de $\mathrm{T}$, a solução é iterativa (Pieri \& Fuchs, 1990).

A fim de avaliar as estimativas de calor sensível pelo método aerodinâmico (H_A), esses valores foram comparados aos valores obtidos pelo método do balanço de energia razão de Bowen (H_B). Esta comparação incluiu o teste t-Student, o erro padrão (EP) e o índice de concordância “d”, de Willmot et al. (1985).

\section{RESULTADOS E DISCUSSÃO}

Na Tabela 1 se apresentam os resultados da análise estatística da comparação do fluxo de calor sensível H obtidos pelo método aerodinâmico (H_A) e pelo método do balanço de energia razão de Bowen (H_B). Segundo a escala proposta por Willmot et al. (1985), observa-se excelente concordância entre os valores de fluxo de calor sensível, obtidos pelo método do balanço de energia razão de Bowen (H_B) e pelo método aerodinâmico (H_A).

Tabela 1. Análise estatística do fluxo de calor sensível obtido pelo método aerodinâmico e pelo método do balanço de energia razão de Bowen

\begin{tabular}{lrcc}
\hline & \multicolumn{1}{c}{ EP $^{1}$} & $\mathbf{d}^{2}$ & Teste $^{\mathbf{3}}$ \\
Todo ciclo & 14,71 & 0,9798 & $\mathrm{~V}$ \\
Período I & 9,78 & 0,9865 & $\mathrm{~V}$ \\
Período II & 13,84 & 0,9859 & $\mathrm{~V}$ \\
Fase de emergência & 16,70 & 0,9870 & $\mathrm{~V}$ \\
Fase vegetativa & 14,38 & 0,9819 & $\mathrm{~V}$ \\
Fase reprodutiva & 6,13 & 0,9739 & $\mathrm{~V}$ \\
Fase de maturação & 12,24 & 0,9745 & $\mathrm{~V}$ \\
\hline
\end{tabular}

1. EP - erro padrão $\left(\mathrm{W} \mathrm{m}^{-2}\right) ; 2 . d$ - índice de concordância de Willmott et al. (1985); 3. V - hipótese verdadeira $(b=1), F$ - hipótese falsa $(b \neq 1)$

O valor do erro padrão (EP) para os valores obtidos durante todo o ciclo fenológico da cultura, foi igual a $14,71 \mathrm{~W} \mathrm{~m}^{-2}$, enquanto para o período em que o solo se encontrava úmido (Período I) foi de $9,78 \mathrm{~W} \mathrm{~m}^{-2}$ e para o Período II (solo mais seco) foi de $13,84 \mathrm{~W} \mathrm{~m}^{-2}$. Já para as fases fenológicas o EP foi de 16,$70 ; 14,38 ; 6,13$ e $12,24 \mathrm{~W} \mathrm{~m}^{-2}$ para a fase de emergência, vegetativa, reprodutiva e de maturação, respectivamente. Os resultados do teste t-Student, com intervalo de confiança de 95\% mostraram que não houve diferença estatística para todo o ciclo nem para os períodos de solo mais úmido (Período I) e mais seco (Período II), bem como para as diferentes fases fenológicas, indicando que os valores de H_A tenderam a ser iguais aos valores de H_B. Ortega-Farias et al. (1996) encontraram resultados de EP um pouco maiores do que o desta pesquisa, quando analisaram todo o período de dados $\left(18,1 \mathrm{~W} \mathrm{~m}^{-2}\right)$, o período de dias úmidos $\left(17,9 \mathrm{~W} \mathrm{~m}^{-2}\right)$ e o período de dias secos $\left(18,3 \mathrm{~W} \mathrm{~m}^{-2}\right)$.

O método aerodinâmico apresentou alta exatidão para a estimativa do fluxo de calor sensível haja vista que tanto para os valores de todo o ciclo quanto para os valores do Período I 
e do Período II, bem como para os valores das fases fenológicas, o índice d apresentou valores acima de 0,97 .

A comparação geral do fluxo de calor sensível obtido pelo método aerodinâmico (H_A) e pelo método do balanço de energia razão de Bowen (H_B), a cada 30 min, é apresentada na Figura 1. A linha de regressão dos pontos ficou bem próxima da linha 1:1, demonstrando que a maior parte dos valores de H_A e H_B ficou próxima dessa linha, com um coeficiente de determinação $\left(\mathrm{R}^{2}\right)$ de 0,926 . Os valores de fluxo de calor sensível mais afastados da linha 1:1 estão acima dos $200 \mathrm{~W} \mathrm{~m}^{-2}$. Ressalta-se que na Figura 1 são utilizados todos os dados de H_B e H_A, durante o período de estudo, ou seja, considerando-se os valores diurnos quanto os noturnos.

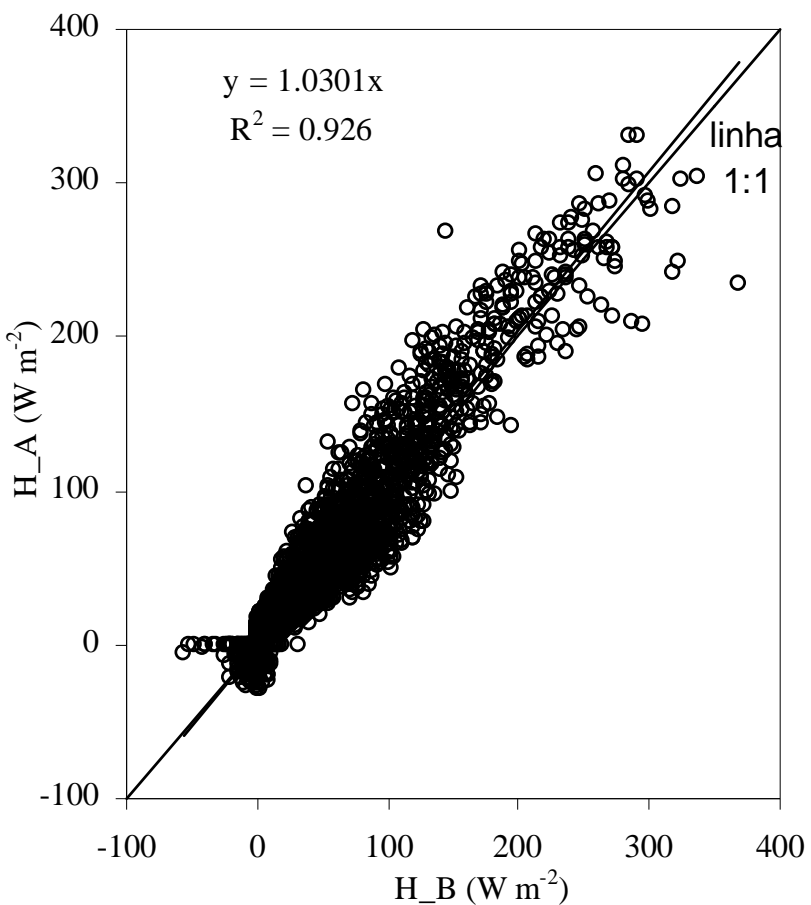

Figura 1. Comparação entre o fluxo de calor sensível obtido pelos métodos da razão de Bowen $\left(H_{-} B\right)$ e aerodinâmico $\left(\mathrm{H}_{-} \mathrm{A}\right)$ para uma cultura de feijão-caupi

Ortega-Farias et al. (1996), utilizaram o método aerodinâmico e o do balanço de energia razão de Bowen para estimar o fluxo de calor sensível em gramínea e encontraram valores do coeficiente de determinação iguais a 0,93 , ou seja, valores muito próximos dos encontrados nesta pesquisa.

Na Figura 2 é apresentada a correlação entre os valores de fluxo de calor sensível obtidos pelo método aerodinâmico (H_A) e pelo método do balanço de energia razão de Bowen (H_B) durante o Período I, ocasião em que o solo se encontrava com umidade volumétrica elevada $\left(0,20 \mathrm{~m}^{3} \mathrm{~m}^{-3}\right)$ (Figura 2A) e durante o Período II, quando o solo se encontrava com baixa umidade volumétrica $\left(0,13 \mathrm{~m}^{3} \mathrm{~m}^{-3}\right)$ (Figura $\left.2 \mathrm{~B}\right)$. Observa-se boa concordância entre H_A e H_B, com valores de $\mathrm{R}^{2}$ de $0,9476 \mathrm{e}$ 0,9612 para os Períodos I e II, respectivamente.

Analisando-se a Figura 2A, Período I, vê-se que a maior parte dos 384 valores de fluxo de calor sensível está próxima da linha 1:1, sendo que desses 384 valores de fluxo de calor sensível, 68\% dos obtidos pelo método aerodinâmico (H_A),
A.

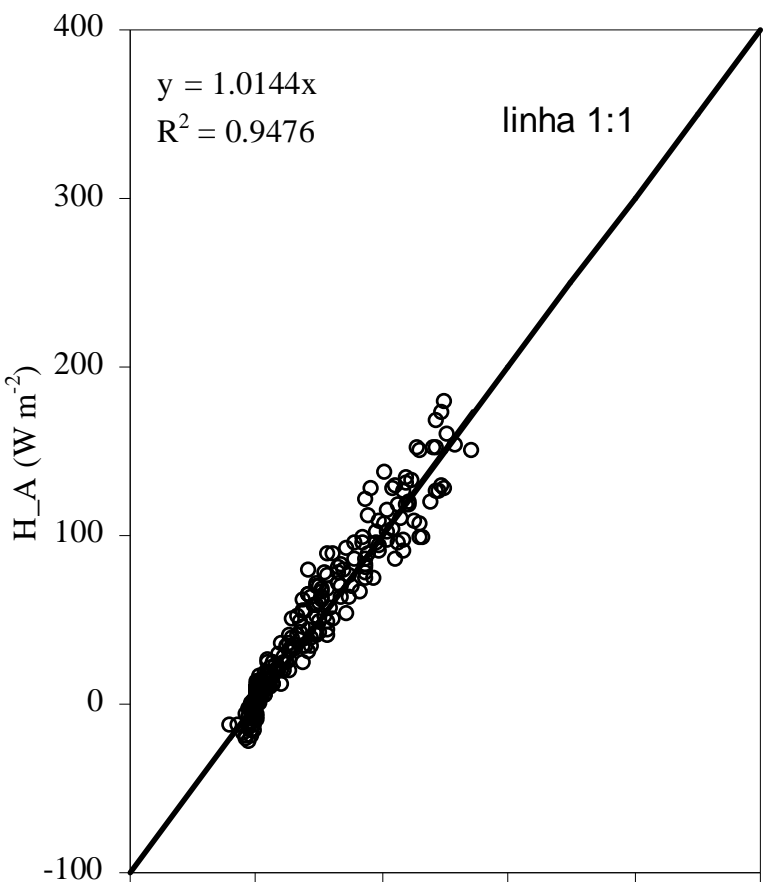

B.

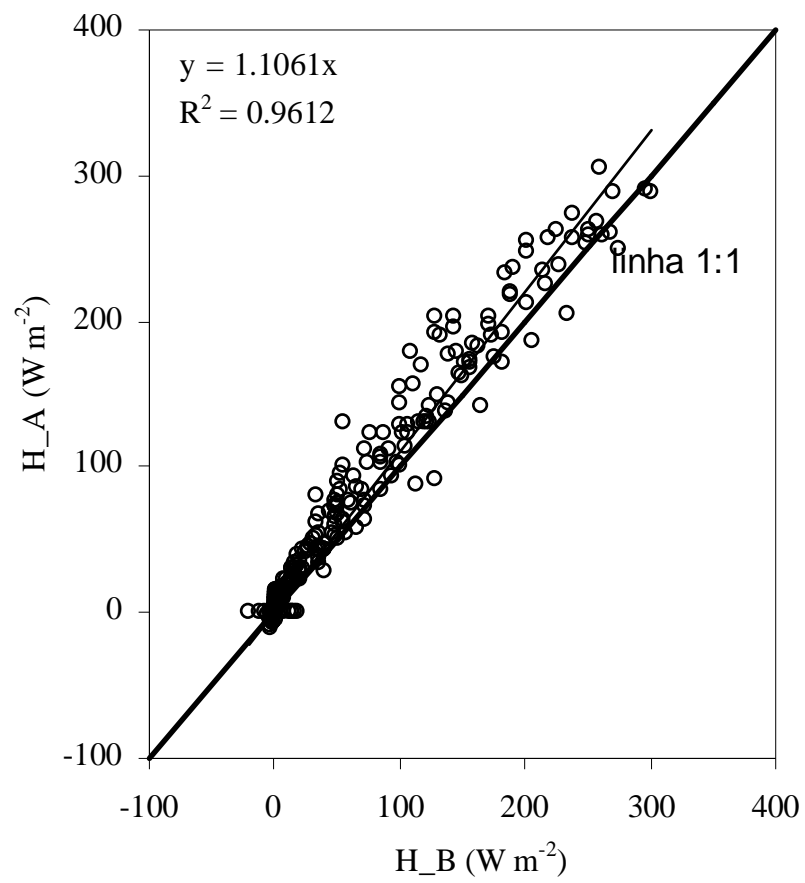

Figura 2. Comparação entre o fluxo de calor sensível obtido pelos métodos aerodinâmico $\left(H \_A\right)$ e do balanço de energia razão de Bowen $\left(H_{-} B\right)$ para uma cultura de feijão-caupi, durante o Períodō I (A) e o Período II (B)

ou seja, 261 valores, são maiores que os obtidos pelo método do balanço de energia razão de Bowen (H_B). Durante o Período II, Figura $2 \mathrm{~B}$ vê-se que, apesar de apresentar um valor de $\mathrm{R}^{2}$ $(0,9612)$ maior do que o do Período I, existe uma dispersão maior dos dados em relação à linha $1: 1$, sendo que $83 \%$ dos valores de fluxo de calor sensível obtido pelo método aerodinâmico (H_A) são maiores que os obtidos pelo método do balanço de energia razão de Bowen (H_B). Observa-se, ainda, que os maiores valores de fluxo de calor sensível são da 
ordem de $180 \mathrm{~W} \mathrm{~m}^{-2}$ e de $300 \mathrm{~W} \mathrm{~m}^{-2}$, para os Períodos I e II, respectivamente. As maiores diferenças encontradas entre H_A e H_B foram de 37,13 e de 76,31 W m², para os Períodos I e II, respectivamente, cujos resultados indicam que durante o Período I ocorreu maior concordância entre H_A e H_B, como já evidenciado pela menor dispersão dos dados em relação à linha 1:1, da Figura 2.

Pereira et al. (1997), mostram que quando a superfície do solo está umedecida, maior parte do saldo de radiação $(\mathrm{Rn})$ será utilizada em calor latente (LE). Se a superfície apresentar restrição hídrica, maior parte de $\mathrm{Rn}$ será utilizada no aquecimento do ar (fluxo de calor sensível). Este fato fica evidenciado nesta pesquisa, pois na Figura 2A (Período I, mais úmido) os valores de fluxo de calor sensível são bem menores que os da Figura 2B (Período II, mais seco), independentemente do método utilizado.

De acordo com Allen et al. (1994), quando a umidade da superfície do solo é menor ocorre um aumento da temperatura da superfície, quando comparado ao solo úmido. Por outro lado, ocorre diminuição da umidade relativa (pressão de vapor) e aumento da velocidade do vento (Tabela 2). Como o método da razão de Bowen utiliza dados de temperatura e de pressão de vapor (equações 4 e 5) e o aerodinâmico utiliza dados de temperatura e de velocidade do vento (equação 6), o aumento da velocidade do vento, a qual diminui a resistência aerodinâmica, e o aumento de temperatura do ar no período mais seco (Período II), fazem com que o fluxo de calor sensível obtido pelo método aerodinâmico (H_A) seja mais elevado que o obtido pelo método da razão de Bowen (H_B).

Polônio \& Soler (2000) usaram os métodos do balanço de energia razão de Bowen e aerodinâmico para determinar o fluxo de calor sensível, em três diferentes áreas na Catalunha, Espanha, cultivadas com alface e alcachofras irrigadas e cevada de sequeiro. Esses autores encontraram que nos períodos de velocidade do vento menor que $3 \mathrm{~m} \mathrm{~s}^{-1}$, existiu uma concordância fraca entre os métodos, o contrário se verificando quando a velocidade do vento era maior que $3 \mathrm{~m} \mathrm{~s}^{-1}$.

Na Tabela 2 são apresentados os valores máximos e mínimos diários da temperatura do ar, da umidade relativa e da velocidade do vento nos dois níveis de medição, durante os períodos I e II.

Os valores máximos de temperatura do ar são, em média, 1,5 ${ }^{\circ} \mathrm{C}$ maiores no período II do que no período I enquanto os valores mínimos são praticamente iguais nos dois períodos, sendo que este comportamento ocorre para os dois níveis de medição. O comportamento da umidade relativa (UR) é diferente da temperatura do ar uma vez que, durante o período I, os valores máximos são praticamente iguais aos do período II e os valores mínimos durante o período I são, em média, 15\% maiores do que no período II. A velocidade do vento (u) apresenta comportamento diferente das variáveis anteriores já que, durante o período I, tanto os valores máximos quanto os mínimos são maiores (em torno de 10\%) do que no período II.

Durante o período mais seco, como já explicado anteriormente, ocorre aumento de temperatura e, consequentemente, diminuição da umidade relativa do ar. Além disso, os próprios componentes do balanço de energia influenciam a velocidade do vento pois. de acordo com Santanna et al. (2008), a intensidade e a direção dos ventos são determinadas pela variação espacial e temporal do balanço de energia na superfície terrestre, que causa variações no campo de pressão atmosférica, gerando os ventos.

A Figura 3 mostra a variação horária do saldo de radiação (Rn) e do fluxo de calor sensível obtido pelo método aerodinâmico (H_A) e pelo método do balanço de energia razão de Bowen (H_B), para o Período I, em dois dias com condições atmosféricas diferentes. O dia 28/03/2002 (Figura 3A) é caracterizado por apresentar alta nebulosidade enquanto o dia

Tabela 2. Valores máximos e mínimos diários da temperatura do ar, da umidade relativa (UR) e da velocidade do vento (u), medidos nos níveis $z_{1}$ e $z_{2}$, durante os Períodos I e ll

\begin{tabular}{|c|c|c|c|c|c|c|c|c|c|c|c|c|}
\hline \multirow{3}{*}{ Data } & \multicolumn{4}{|c|}{ Temperatura do ar $\left({ }^{\circ} \mathrm{C}\right)$} & \multicolumn{4}{|c|}{ UR (\%) } & \multicolumn{4}{|c|}{$u\left(\mathrm{~m} \mathrm{~s}^{-1}\right)$} \\
\hline & \multicolumn{2}{|c|}{$z_{1}=0,35 \mathrm{~m}$} & \multicolumn{2}{|c|}{$z_{2}=1,05 \mathrm{~m}$} & \multicolumn{2}{|c|}{$z_{1}=0,35 \mathrm{~m}$} & \multicolumn{2}{|c|}{$z_{2}=1,05 \mathrm{~m}$} & \multicolumn{2}{|c|}{$z_{1}=0,35 \mathrm{~m}$} & \multicolumn{2}{|c|}{$z_{2}=1,05 \mathrm{~m}$} \\
\hline & Máx & Mín & Máx & Mín & Máx & Mín & Máx & Mín & Máx & Mín & Máx & Mín \\
\hline \multicolumn{13}{|c|}{ "Período l" } \\
\hline $23 / 03 / 2002$ & 27,23 & 20,59 & 26,59 & 20,49 & 100,0 & 75,2 & 99,1 & 73,5 & 3,19 & 0,55 & 4,25 & 0,45 \\
\hline $24 / 03 / 2002$ & 26,46 & 20,14 & 25,76 & 20,16 & 100,0 & 81,0 & 99,5 & 78,5 & 2,61 & 0,53 & 3,33 & 0,45 \\
\hline $25 / 03 / 2002$ & 27,96 & 19,77 & 27,34 & 19,80 & 99,9 & 63,3 & 99,1 & 61,2 & 2,79 & 1,09 & 3,56 & 1,33 \\
\hline $26 / 03 / 2002$ & 27,86 & 19,68 & 27,09 & 19,71 & 100,0 & 71,1 & 99,1 & 70,5 & 2,82 & 1,09 & 3,80 & 0,56 \\
\hline $27 / 03 / 2002$ & 27,27 & 20,82 & 27,01 & 20,78 & 99,6 & 60,4 & 98,6 & 57,6 & 2,73 & 1,51 & 3,74 & 1,99 \\
\hline $28 / 03 / 2002$ & 26,06 & 20,37 & 25,47 & 20,39 & 99,9 & 80,8 & 98,6 & 79,7 & 2,99 & 1,13 & 4,18 & 1,38 \\
\hline $29 / 03 / 2002$ & 27,69 & 19,68 & 27,22 & 19,70 & 100,0 & 63,7 & 99,0 & 61,2 & 3,26 & 1,77 & 4,57 & 1,98 \\
\hline $30 / 03 / 2002$ & 29,07 & 20,56 & 28,37 & 20,51 & 100,0 & 52,6 & 99,0 & 51,2 & 2,86 & 1,72 & 3,99 & 0,45 \\
\hline Média & 27,45 & 20,20 & 26,86 & 20,19 & 99,9 & 68,5 & 99,0 & 66,7 & 2,91 & 1,17 & 3,93 & 1,07 \\
\hline \multicolumn{13}{|c|}{ "Período II" } \\
\hline $08 / 04 / 2002$ & 30,84 & 19,38 & 29,63 & 19,46 & 99,1 & 47,5 & 97,1 & 48,3 & 2,53 & 1,18 & 3,37 & 1,54 \\
\hline $09 / 04 / 2002$ & 30,51 & 20,64 & 29,22 & 20,52 & 99,5 & 49,8 & 97,8 & 51,6 & 2,66 & 1,19 & 3,49 & 0,45 \\
\hline $10 / 04 / 2002$ & 29,25 & 20,58 & 28,17 & 20,50 & 100,0 & 64,0 & 98,7 & 64,8 & 2,79 & 0,93 & 3,66 & 0,87 \\
\hline $11 / 04 / 2002$ & 29,84 & 20,77 & 28,71 & 20,75 & 100,0 & 54,4 & 99,3 & 54,6 & 2,88 & 1,39 & 3,78 & 1,34 \\
\hline $12 / 04 / 2002$ & 25,87 & 20,98 & 25,29 & 21,05 & 100,0 & 72,3 & 98,6 & 71,8 & 2,63 & 0,45 & 3,59 & 0,45 \\
\hline $13 / 04 / 2002$ & 30,11 & 18,31 & 29,11 & 19,36 & 100,0 & 56,4 & 98,6 & 57,0 & 2,54 & 0,45 & 3,44 & 0,45 \\
\hline $14 / 04 / 2002$ & 27,56 & 20,67 & 26,89 & 20,62 & 100,0 & 66,8 & 99,0 & 65,8 & 2,67 & 1,14 & 3,70 & 1,43 \\
\hline $15 / 04 / 2002$ & 29,32 & 20,11 & 28,35 & 20,03 & 100,0 & 56,0 & 99,3 & 56,1 & 2,47 & 0,96 & 3,39 & 1,42 \\
\hline Média & 29,16 & 20,18 & 28,17 & 20,29 & 99,8 & 58,4 & 98,6 & 58,7 & 2,65 & 0,96 & 3,55 & 0,99 \\
\hline
\end{tabular}


29/03/2002 (Figura 3B) se caracteriza por apresentar condições de céu praticamente claro. A condição de nebulosidade pode ser inferida dos dados do saldo de radiação $(\mathrm{Rn})$.

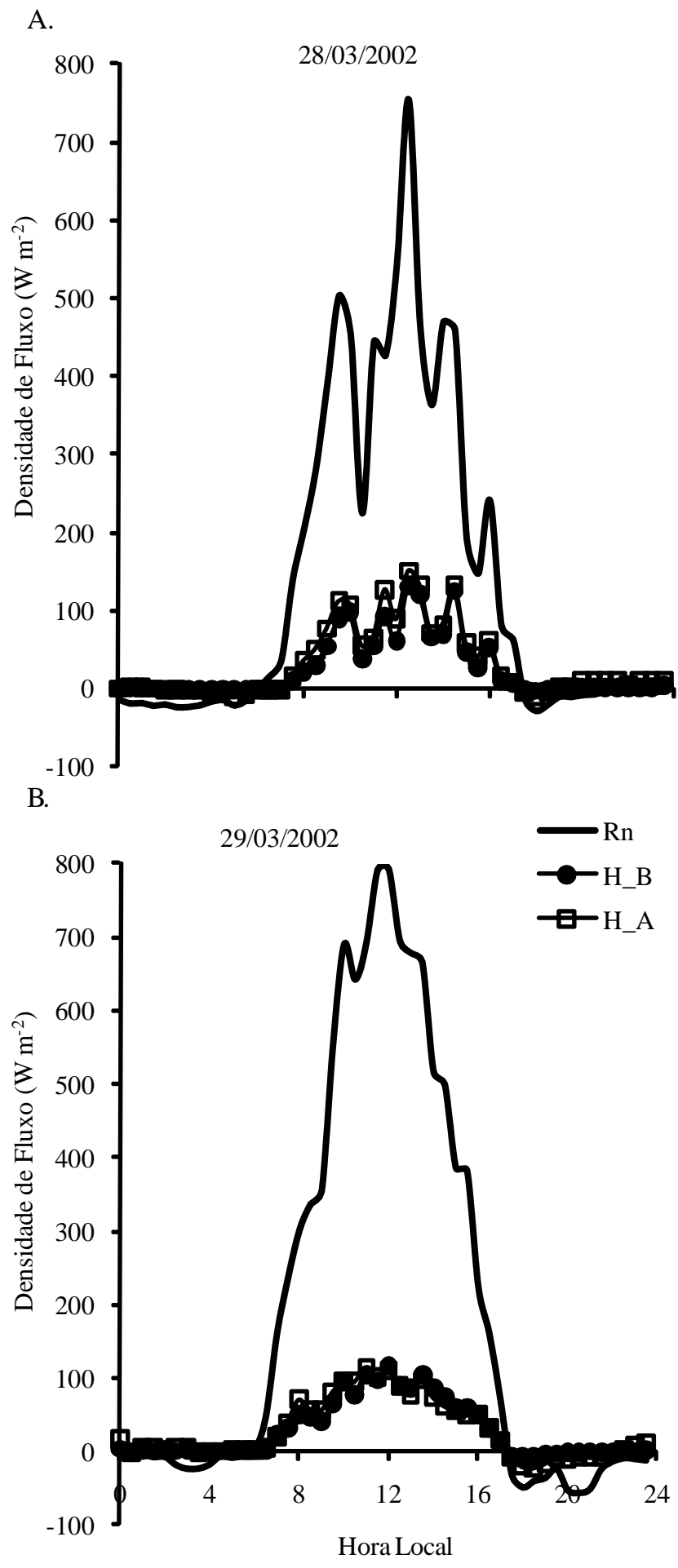

Figura 3. Variação horária do sal do de radiação (Rn) e do fluxo de calor sensível obtido pelos métodos do balanço de energia razão de Bowen $\left(H_{-} B\right)$ e aerodinâmico $\left(H_{-} A\right)$, nos dias 28/03/2002 (A) e 29/03/2002 (B) (Período I)

Observa-se, na Figura 3A, boa concordância entre H_A e H_B, sendo que existe uma pequena discordância das 11 h e 30 min às 12 h e 30 min. Os maiores valores de H_A e H_B estão em torno de 150,0 e $130,0 \mathrm{~W} \mathrm{~m}^{-2}$, respectivamente, em que a maior diferença entre H_A e H_B foi de $35,3 \mathrm{~W} \mathrm{~m}^{-2}$. Constatase, na Figura 3B, excelente concordância entre H_A e H_B para todas as horas do dia, sendo os maiores valores de H_A e H_B girando em torno de 110,0 e $116,0 \mathrm{~W} \mathrm{~m}^{-2}$, respectivamente, sendo a maior diferença entre H_A e H_B de $21,0 \mathrm{~W} \mathrm{~m}^{-2}$.

A nebulosidade ocorrida no dia 28/03/2002 causou uma variação do saldo de radiação e exerceu efeito direto em H_B; contudo, os valores de H_A, que são independentes das medições do saldo de radiação, mostraram as mesmas flutuações, o que torna esses resultados altamente significativos; o mesmo comportamento foi encontrado por Ortega-Farias et al. (1996), num campo cultivado com gramíneas, no Oregon (EUA).

A Figura 4 mostra a variação horária do saldo de radiação $(\mathrm{Rn})$ e do fluxo de calor sensível obtido pelo método aerodinâmico (H_A) e pelo método do balanço de energia razão de Bowen (H_B), para o Período II. Os dias 09/04/2002 (Figura 4A) e 13/04/2002 (Figura 4B) se caracterizam por apresentarem condições de céu praticamente claro.

Observa-se, na Figura 4A, a ocorrência de uma boa concordância entre H_A e H_B, em que as maiores discordâncias ocorrem das 8 às 12 h, e as maiores concordâncias ocorrem após as $16 \mathrm{~h}$. Os maiores valores de H_A e H_B estão em torno de 305,0 e 297,0 W m ${ }^{-2}$, respectivamente, sendo a maior diferença entre H_A e H_B de $75,0 \mathrm{~W} \mathrm{~m}^{2}$. Observa-se, na Figura 4B como na Figura 4A, que as maiores discordâncias entre H_A e H_B ocorrem das 8 às 12 h, enquanto as maiores concordâncias ocorrem após as 16 h, sendo os maiores valores de H_A e H_B de 275,0 e 239,0 W m ${ }^{-2}$, respectivamente, enquanto a maior diferença entre H_A e H_B foi de $69,0 \mathrm{~W} \mathrm{~m}^{-2}$.

A partição do saldo de radiação entre os fluxos de calor latente e de calor sensível sobre a cultura do feijão-caupi, foi afetada pelas condições atmosféricas e de umidade volumétrica do solo, cujos resultados indicaram que em ambos os métodos (aerodinâmico e do balanço de energia razão de Bowen), o fluxo de calor sensível foi maior quando existia um baixo conteúdo de água no solo (Período II) do que quando o conteúdo de água no solo era elevado (Período I) (Figuras 3 e 4). Quando o solo se encontrava com conteúdo de água elevado (Período I), os valores médios de H_A e de H_B foram 19 e $18 \%$ da energia disponível $(\mathrm{Rn}-\mathrm{G})$, respectivamente e, quando o conteúdo de água no solo foi de $0,13 \mathrm{~m}^{3} \mathrm{~m}^{-3}$ (Período II), os valores médios de H_A e de H_B foram de 36 e de $31 \%$, respectivamente.

Outros autores encontraram resultados semelhantes. Por exemplo, Neves et al. (2008) determinaram os componentes do balanço de energia em feijão-caupi, em Tracuateua-PA, sob condições de deficiência hídrica e encontraram que $61 \%$ do Rn foram usados como H; já San José et al. (2003), mediram os componentes do balanço de energia em feijão-caupi na Venezuela, sem déficit hídrico e encontraram que em torno de $27 \%$ do $\mathrm{Rn}$ foram usados como $\mathrm{H}$. 


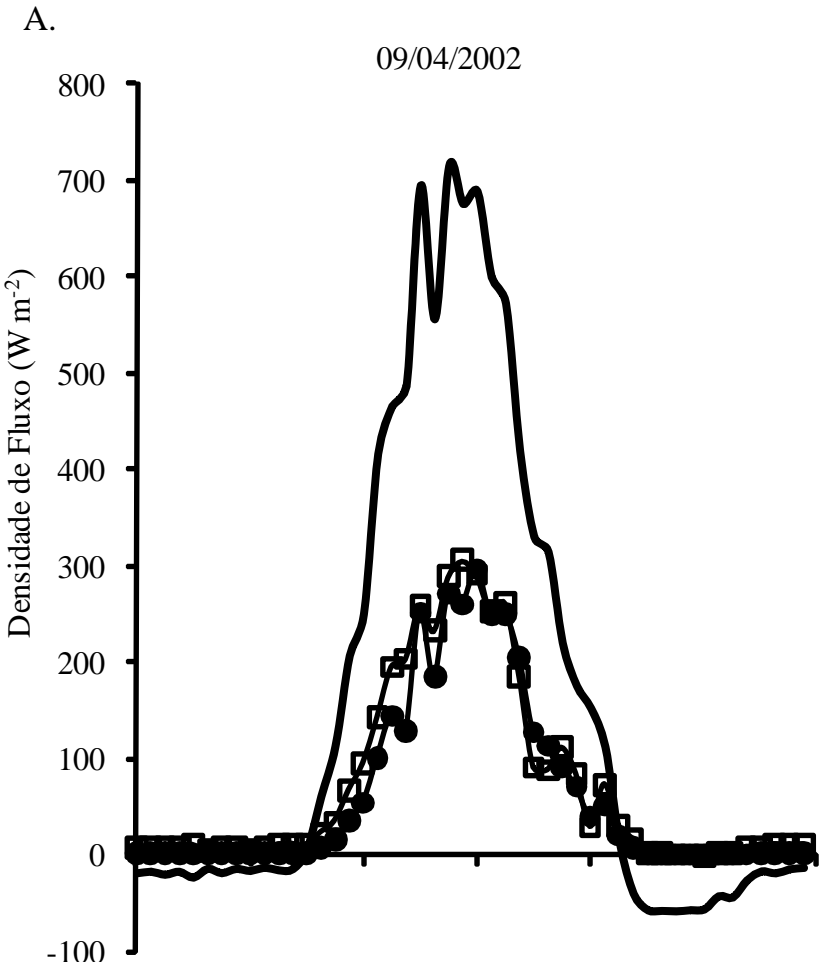

B.

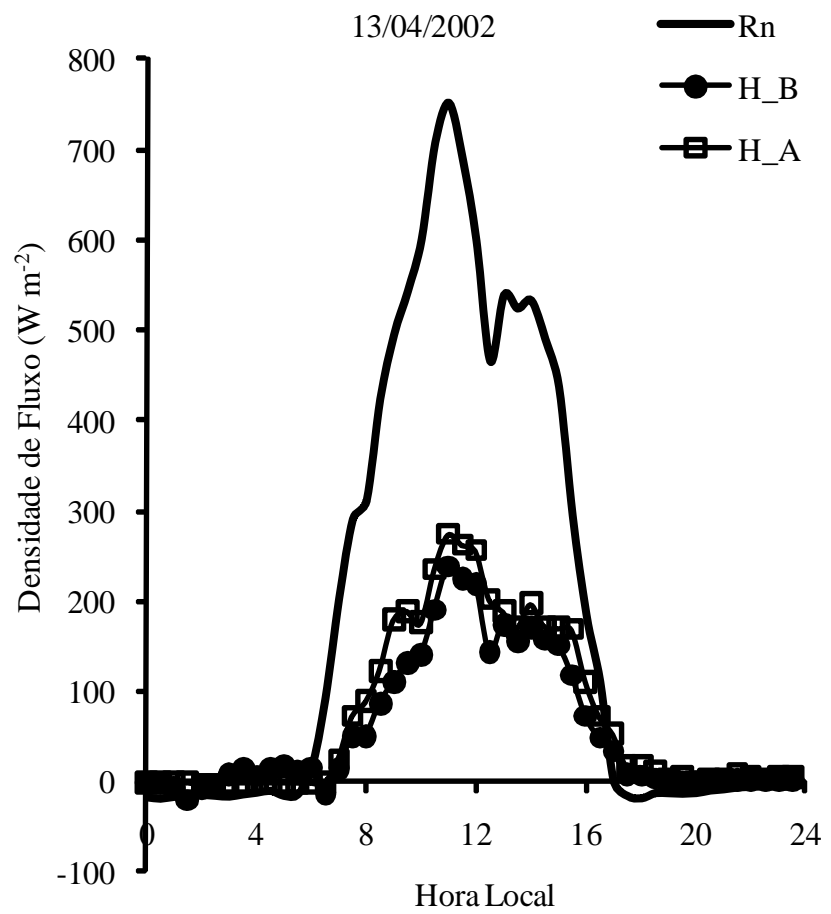

Figura 4. Variação horária do sal do de radiação $(\mathrm{Rn})$ e do fluxo de calor sensível obtido pelos métodos do balanço de energia razão de Bowen $\left(H \_B\right)$ e aerodinâmico $\left(H \_A\right)$, nos dias 09/04/2002 (A) e 13/0̄4/2002 (B) (Período II)

\section{CONClusÕes}

1. O método aerodinâmico pode estimar as variações horárias do fluxo de calor sensível, de forma tão boa quanto o método do balanço de energia razão de Bowen, independentemente das condições de umidade do solo e das condições atmosféricas.
2. O método aerodinâmico pode ser usado, juntamente com medidas do saldo de radiação e do fluxo de calor no solo, para a estimativa do fluxo de calor latente e, consequentemente, da evapotranspiração da cultura do feijão-caupi.

\section{LITERATURA CITADA}

Allen, S. J.; Wallace, J. S.; Gash, J. H. C.; Sivakumar, M. V. K. Measurements of albedo variation over natural vegetation in the Sahel. International Journal of Climatology, v.14, p.625636, 1994.

Azevedo, P. V. de; Souza, C. B.; Silva, B. B. da; Silva, V. de P. R. da. Water requirements of pineapple crop grown in a tropical environment, Brazil. Agricultural Water Management, v.88, p.201-208, 2007.

Bergamaschi, H.; Ometto, J. C.; Vieira, H. J.; Angelocci, L. R.; Libardi, P. L. Deficiência hídrica em feijoeiro. II. Balanço de Energia. Pesquisa Agropecuária Brasileira, v.23, p.745-757, 1988.

Bezerra, J. R. C.; Azevedo, P. V. de; Silva, B. B. da; J. M. Evapotranspiração e coeficiente de cultivo do algodoeiro BRS-200 Marrom, irrigado. Revista Brasileira de Engenharia Agrícola e Ambiental, v.14, p.625-632, 2010.

Bidlake, W. R.; Woodham, W. M.; Lopez, M. A. Evapotranspiration from areas of native vegetation in westcentral Florida. U.S. Geological Survey water-supply paper 2430. 1986. 35p.

Brutsaert, W. Evaporation into the atmosphere. Reidel: Dordrecht, 1982. 299p.

Dyer, A. J.; Hicks, B. B. Flux-gradient relationships in the constant flux layer. Quarterly Journal of the Royal Meteorology Society, v.96, p.715-721, 1970.

EMBRAPA - Empresa Brasileira de Pesquisa Agropecuária. Centro Nacional de Pesquisa de Solos. Sistema Brasileiro de Classificação de Solos. 1.ed. Rio de Janeiro: Embrapa Solos, 2006. 306p.

Gentine, P.; Entekhabi, D.; Chehbouni, A.; Boulet, G.; Duchemin, B. Analysis of evaporative fraction diurnal behaviour. Agricultural and Forest Meteorology, v.143, p.13-29, 2007.

Kolle, O. Long-term comparison of energy flux calculation methods over an agricultural field. Physics and Chemistry of The Earth, v.21, p.111-117, 1996.

Kustas, W. P.; Pruegger, J. H.; Hatfield, J. L.; Ramalingam, K.; Hipps, L. E. Variability in soil heat flux from a mesquite dune site. Agricultural and Forest Meteorology, v.103, p.249-264, 2000.

Lima, J. R. de S. Balanço hídrico e de energia em solo cultivado e sem vegetação, para as condições do Brejo Paraibano. Recife: UFPE, 2004. 167p. Tese Doutorado

Lima, J. R. de S.; Antonino, A. C. D.; Soares, W.A.; Borges, E.; Silva, I. F.; Lira, C. A. B. O. Balanço de energia em um solo cultivado com feijão-caupi no brejo paraibano. Revista Brasileira de EngenhariaAgrícola eAmbiental, v.9, p.527-534, 2005.

Neves, L. O.; Costa, J. M. N.; Andrade, V. M.; Lôla, A. C.; Ferreira, W. P. Balanço de energia em uma cultura de feijãocaupi (Vigna unguiculata L.) no estado do Pará. Revista Brasileira de Agrometeorologia, v.16, p.21-30, 2008. 
Oliveira, I. A.; Lima, J. R. S.; Silva, I. F.; Antonino, A. C. D.; Gouveia Neto, G. C.; Lira, C. A. B. O. Balanço de energia em mamona cultivada em condições de sequeiro no Brejo Paraibano. Revista Brasileira de Ciências Agrárias, v.4, p.185191, 2009.

Ortega-Farias, S. O.; Cuenca, R. H.; Ek, M. Daytime variation of sensible heat flux estimated by the bulk aerodynamic method over a grass canopy. Agricultural and Forest Meteorology, v.81, p.131-143, 1996.

Paulson, C. A. The mathematical representation of wind speed and temperature profiles in the unstable atmospheric surface layer. Journal of Applied Meteorology, v.9, p.857-861, 1970.

Pereira, A. R.; Villa Nova, N. A.; Sediyama, G. C. Evapo(transpi)ração. Piracicaba: FEALQ, 1997. 183p.

Pieri, P.; Fuchs, M. Comparison of Bowen ratio and aerodynamic estimates of evapotranspiration. Agricultural and Forest Meteorology, v.49, p.243-256, 1990.

Polonio D.; Soler, M. R. Surface fluxes estimation over agricultural areas. Comparison of methods and the effects of land surface inhomogeneity. Theoretical and Applied Climatology, v.67, p.65-79, 2000.

Rana, G.; Katerji, N. Measurement and estimation of actual evapotranspiration in the field under Mediterranean climate: a review. European Journal of Agronomy, v.13, p.125-153, 2000.

Rolim, G. S.; Escobedo, J. F.; Oliveira, A. P. Validation of the Deardoff model for estimating energy balance components for a sugarcane crop. Scientia Agricola, v.65, p.325-334, 2008.

San José, J. J.; Bracho, R.; Montes, R.; Nikonova, N. Comparative energy exchange from cowpeas (Vigna unguiculata (L) Walp cvs. TC-9-6 and M-28-6-6) with differences in canopy architectures and growth durations at the Orinoco llanos. Agricultural and Forest Meteorology, v.116, p.197-219, 2003.
Santanna, F. B.; Arruda, P. H. Z.; Farias, J. L. B.; Nogueira, J. S. Estudo preliminar da velocidade e direção dos ventos, em Cuiabá, MT, Brasil. Revista Brasileira de Agrometeorologia, v.16, p.175-180, 2008.

Silva, T. G. F.; Moura, M. S. B.; Zolnier, S.; Soares, J. M.; Souza, L. S. B.; Brandão, E. O. Variação do balanço de radiação e de energia da cana-de-açúcar irrigada no semiárido brasileiro. Revista Brasileira de Engenharia Agrícola e Ambiental, v.15, p.139-147, 2011.

Souza, C. B.; Silva, B. B. da; Azevedo, P. V. de; Silva, V. de P. R. da. Fluxos de energia e desenvolvimento da cultura do abacaxizeiro. Revista Brasileira de Engenharia Agrícola e Ambiental, v.12, p.400-407, 2008.

Teixeira, A. H. C.; Bastiaanssen, W. G. M.; Moura, M. S. B.; Soares, J. M.; Ahmad, M. D.; Bos, M. G. Energy and water balance measurements for water productivity analysis in irrigated mango trees, Northeast Brazil. Agricultural and Forest Meteorology, v.148, p.1524-1537, 2008.

Vries, D. A. de. Thermal properties of soils. In: van Wijk, W. R. Physics of Plant Environment. Amsterdam: North-Holland, 1966. p.210-233.

Willmott, C. J.; Ackleson, S. G.; Davis, R. E.; Feddema, J. J.; Klink, K. M.; Legates, D. R.; O’Donnell, J.; Rowe, C. M. Statistics for the evaluation and comparison of models. Journal of Geophysical Research, v.90, p.8995-9005, 1985.

Zhang, B. Z.; Kang, S. Z.; Zhang, L.; Du, T. S.; Li, S. E.; Yang, $X$. Y. Estimation of seasonal crop water consumption in a vineyard using Bowen ratio-energy balance method. Hydrological Processes, v.21, p.3635-3641. 2007.

Zhou, L.; Zhou, G. Measurement and modelling of evapotranspiration over a reed (Phragmites australis) marsh in Northeast China. Journal of Hydrology, v.372, p.41-47, 2009. 